\title{
EDITORIAL
}

\section{Signalling and transcriptional regulation in inflammatory and immune cells: importance in lung biology and disease}

\author{
K.F. Chung and I.M. Adcock
}

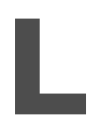

iving cells respond to their external environment and to signals from other cells by transducing these signals to different parts of the cell, particularly to the nucleus. This idea was initiated by RALL and SUTHERLAND [1], with the concept of cyclic adenosine monophosphate acting as a second messenger to mediate the action of the first messenger that arrived at the cell surface. Much of cell biology is now focused on dissecting out signalling pathways from the cell membrane to the nucleus and also to other parts of the cell, and on how the ultimate cellular response will be determined by the signalling pathways activated. Many signalling pathways regulate cell growth, cell survival, cell differentiation, cell migration, cell metabolism and transcriptional regulation of inflammatory genes, and it will not be surprising that derangement of these pathways can form the basis of disease processes involved in inflammation.

Indeed, for any physiological system, signalling pathways must have adequate controlling steps; with the complexity of many signalling networks in different parts of the cell, it is now important to develop high-resolution maps of signalling networks in the living cell [2]. This determination of spatial and temporal organisation of signal transduction will be greatly helped by the introduction of techniques such as the development of designer fluorescence resonance energy transfer, which is capable of detecting specific protein-protein interactions defining signalling pathways in live cells [3]. In terms of disease, the presence of a mediator is not the end of the line, but how this transduces the signal has to be determined. Can disease occur as a result of excessive or a lack of these signalling pathways? The answer is obviously yes, and interference with these signalling pathways can lead to therapeutics. Existing drugs such as $\beta$-adrenergic agonists and corticosteroids, which are used for the treatment of asthma and chronic obstructive pulmonary disease, transduce their effects by targeting many signalling pathways. Similarly, pro-inflammatory insults and oxidative stress can impact on signalling pathways. The development of the selective kinase inhibitor, imatinib, for the treatment of cancer or hypereosinophilic syndrome attests to the potential for new drugs that selectively target specific signalling pathways. However, this area of drug

Airways Disease Section, National Heart and Lung Institute, Imperial College, London, UK.

CORRESPONDENCE: K.F. Chung, National Heart and Lung Institute, Dovehouse St, London SW3 6LY, UK. Fax: 44 2073518126. E-mail: f.chung@imperial.ac.uk development is difficult because there are $>500$ protein kinases in the human genome with relatively conserved active sites [4].

Beginning in this issue of the European Respiratory Journal (ERJ), a series of articles will be published, detailing how immune and inflammatory cells respond to external stimuli to transduce the initial signal, and enhance and perpetuate the inflammatory response, and how these signals can be modified in disease and by therapies, with specific relevance to respiratory conditions. The signalling pathway can be initiated by receptor or nonreceptor activation. Receptor activation can often be linked to the activation of secondary messengers, such as the mitogen-activated protein kinases (MAPK) or phosphoinositol-3 kinase, which could lead to downstream activation of transcription factors, such as nuclear factor (NF)$\kappa \mathrm{B}$ or activator protein-1, leading to activation of nuclear signals to enhance the transcription of key mediator proteins. This may be one of the pathways by which certain cell types could be excessively producing cytokines/chemokines. Gene transcription is now seen to be a complex process, involving the recruitment of transcriptional complexes that control the degree of histone modifications and the remodelling of the tightly compacted chromatin structure. Gene transcription and production of mRNA alone is not the sole regulator of mRNA expression. For example, the expression of many chemokines is regulated by the ability of p38 MAPK to modify mRNA stability. The instability of many pro-inflammatory mRNAs is critical in maintaining low mRNA levels until an appropriate stimulus arrives, and allows gene expression to be rapidly switched off as the inflammatory response resolves. mRNA stability is regulated by proteins that bind to adenosine-/ uridine-rich elements (AREs) within the 3' untranslated regions of pro-inflammatory mRNAs. One such protein, tristetraprolin (TTP), restrains the expression of tumour necrosis factor (TNF)- $\alpha$; TTP-knockout mice overexpress TNF- $\alpha$ with subsequent severe inflammatory disease.

Some important signalling units are provided for the ERJ readers to illustrate some of the interesting features by which certain signals can be transduced. NF- $\kappa \mathrm{B}$, as mentioned previously, is one of the key signalling molecules in immune and inflammatory cells, and plays a role in the expression of most inflammatory genes and, additionally, in the regulation of apoptosis [5]. Signalling by NF- $\mathrm{kB}$ involves phosphorylation and subsequent ubiquitin targeting for proteosomal 
degradation of inhibitor (I) $\kappa \mathrm{B} \alpha$ within the cytosol, followed by translocation of NF- $\kappa B$ into the nucleus. NF- $\kappa B$ activation of $\mathrm{I} \kappa \mathrm{B} \alpha$ transcription represents a delayed negative feedback loop. Transcription of NF- $\kappa \mathrm{B}$ target genes depends upon persistent oscillations of NF- $\kappa \mathrm{B}$, involving cycles of RelA phosphorylation and dephosphorylation. Thus, the functional consequences of NF- $\kappa \mathrm{B}$ signalling may depend on number, period and amplitude of oscillations. Another aspect of NF- $\kappa B$ signalling is the association of histone deacetylase (HDAC) with inactive $\mathrm{p} 65$ component of NF- $\mathrm{KB}$, and the role played by

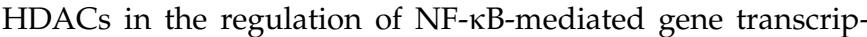
tion without altering DNA binding. Indeed, p65 and other transcription factors, such as GATA, are also targets for acetylation by HDACs, thereby modifying their transcriptional activity.

The MAPK pathways represent key routes through which extracellular stimuli are transmitted into nuclear responses [6]. The extracellular-regulated MAPK (ERK) pathway responds to mitogenic stimuli, whereas the p38 and c-jun N-terminal kinase pathways are activated by cellular stresses. Upon reaching the nucleus, the terminal MAPKs in these cascades phosphorylate a number of key transcription factors including c-Jun, activating transcription factor-2 and Ets (E26)-like kinase (Elk)-1. Recently, Elk-1 has been shown to also be a target of the SUMO modification pathway. Whereas phosphorylation by MAPKs is an activating event, SUMO modification inhibits the activity of Elk-1. Activation of Elk-1 by the ERK pathway also leads to the loss of SUMO modification, providing an elegant activating switch that also removes the inhibitory SUMO modification. Sumoylation of many transcription factors occurs and generally correlates with transcriptional repression due to recruitment of HDACs. There is a more complex regulatory interplay between these two pathways.

The MAPK p38 pathway is activated by pro-inflammatory stimuli. One of the functions of the p38 pathway is to stabilise pro-inflammatory mRNAs. In conjunction with well-characterised transcriptional mechanisms, mRNA stabilisation by $\mathrm{p} 38$ can bring about rapid, dramatic and short-lived changes in gene expression. MAPK p38 regulates mRNA stability by altering the expression or function of one or more ARE binding proteins. Indeed, the p38 pathway regulates TTP (see above) at multiple levels.

Finally, information about the signalling pathways underlying the innate immune system, which is the first line of defence against invading pathogens and may also be used by inflammatory proteins, indicates how nature can use the diverse signalling units for maintaining specificity of the inflammatory response [7]. First, there are up to 11 toll-like receptors (TLR) involved, and, secondly, there are also many adaptor family members including MyD88, Mal, Trif and Tram that tailor the signalling pathways activated by TLRs. For example, TLR3 uses Trif to activate its major transcription factor target, interferon regulatory factor (IRF)-3, whereas TLR4 can use all four adaptors to activate both NF- $\kappa B$ and
IRF-3. Identification of the receptors SIGIRR and ST2 as negative regulators of TLR signalling pathways indicates a high level of complexity in the control of signalling from these receptors.

The focus of the first article in this series will be on the posttranscriptional regulation of chemokines, which may play an important role in lung inflammation and allergy [8]. Following this, there will be reviews on the signal pathways regulating Thelper type- 2 cells, the growth factors and oxidative stress regulation of transduction mechanisms, MAPK pathways, and the pathways of corticosteroid actions and of $\beta$-adrenergic receptor stimulation.

In a recent survey, it appeared that respiratory clinicians are not interested in cell and molecular biology, and one of the reasons provided to explain this attitude was that clinicians deal with diseased organ systems which are more complex than just individual cells or molecules [9]. However, this cannot be the entire reason; more likely, clinicians do not yet appreciate the impact that discoveries about signalling transduction mechanisms may have on their daily practice. It is hoped that this series will stimulate the healthy interest that clinicians should keep in this rapidly advancing field of cell signalling networks, which will transduce into an understanding of pathogenesis and better treatments.

\section{REFERENCES}

1 Rall TW, Sutherland EW. Formation of a cyclic adenine ribonucleotide by tissue particles. J Biol Chem 1958; 232: 1065-1076.

2 White MA, Anderson RG. Signaling networks in living cells. Annu Rev Pharmacol Toxicol 2005; 45: 587-603.

3 Sekar RB, Periasamy A. Fluorescence resonance energy transfer (FRET) microscopy imaging of live cell protein localizations. J Cell Biol 2003; 160: 629-633.

4 Cohen MS, Zhang C, Shokat KM, Taunton J. Structural bioinformatics-based design of selective, irreversible kinase inhibitors. Science 2005; 308: 1318-1321.

5 Chen LF, Greene WC. Shaping the nuclear action of NFkappaB. Nat Rev Mol Cell Biol 2004; 5: 392-401.

6 Kyriakis JM, Avruch J. Mammalian mitogen-activated protein kinase signal transduction pathways activated by stress and inflammation. Physiol Rev 2001; 81: 807-869.

7 Dunne A, O'Neill LA. Adaptor usage and Toll-like receptor signaling specificity. FEBS Lett 2005; 579: 3330-3335.

8 Fan J-S, Heller NM, Gorospe M, Atasoy U, Stellato C. The role of post-transcriptional regulation in chemokine gene expression in inflammation and allergy. Eur Respir J 2005; 26: 933-947.

9 Macklem PT. Is cell and molecular biology divorcing from clinical practice? Am J Respir Crit Care Med 2003; 167: 1164-1165. 